\title{
Seismic evolution of low/intermediate mass PMS stars (Research Note)
}

\author{
F. J. G. Pinheiro
}

Centro de Astrofísica da Universidade do Porto, Rua da Estrelas, 4150-762 Porto, Portugal

e-mail: fjgp@astro.up.pt

Received 19 January 2007 / Accepted 31 October 2007

\section{ABSTRACT}

\begin{abstract}
This article presents a study of the evolution of the internal structure and seismic properties expected for low/intermediate mass Pre-Main Sequence (PMS) stars. Seismic and non-seismic properties of PMS stars were analysed. This was done using 0.8 to $4.4 M_{\odot}$ stellar models at stages ranging from the end of the Hayashi track up to the Zero-Age Main-Sequence (ZAMS). This research concludes that, for intermediate-mass stars $\left(M>1.3 M_{\odot}\right)$, diagrams comparing the effective temperature $\left(T_{\text {eff }}\right)$ against the small separation can provide an alternative to Christensen-Dalsgaard (C-D) diagrams. The impact of the metal abundance of intermediate mass stars $\left(2.5-4.4 M_{\odot}\right)$ has over their seismic properties is also evaluated.
\end{abstract}

Key words. stars: evolution - stars: interiors - stars: oscillations - stars: pre-main sequence

\section{Introduction}

The study of the Sun's seismic characteristics allows a better understanding of its internal structure (e.g. Antia 2005). Yet, for other stars it is only possible to observe pulsations associated with low degree spherical harmonics, constraining the results that one can obtain (Bedding \& Kjeldsen 2003). Still, asteroseismic techniques can be used to infer the internal structure of solar-type stars (e.g. Monteiro et al. 2002)

The seismic study of Pre-Main Sequence (PMS) stars (e.g. Breger 1972; Marconi \& Palla 1998; Ripepi \& Marconi 2004) has been mainly focused on objects located inside the PMS Instability Strip (IS). However, in terms of the location and size of the outer convective regions, low mass PMS stars, located near the Zero Age Main Sequence (ZAMS), resemble solar-type stars. Therefore they may be expected to oscillate.

Not much work has been done regarding the study of PMS solar-type pulsations (Samadi et al. 2005; Pinheiro et al. 2006). This research goes beyond the study of the seismic properties of young stars initiated by Pinheiro et al. (2006) by extending Monteiro et al.'s (2002) analysis to PMS stars. By taking into account the evolution of the seismic properties of young stars we evaluate the possibility of testing PMS evolutionary models through the use of solar-type pulsations.

\section{Solar-type pulsations}

The Sun, amongst other solar-type stars, displays a type of oscillation in which pressure acts as a restoring force. These $p$-mode pulsations, known as solar-type oscillations, are stochastically driven by the outer convective layers. The power spectrum of solar-type pulsators presents two typical frequency separations, known as the large $\left(\Delta v_{n, l}\right)$ and small $\left(\delta v_{n, l}\right)$ separation. The first one corresponds to the difference between frequencies associated with oscillations of the same degree $(l)$ and consecutive overtone $(n)$, while the latter corresponds to the difference between frequencies $v_{n-1, l+2}$ and $v_{n, l}$. Tassoul (1980) showed that in the asymptotic regime $(n \gg l)$ these two separations can be written as:

$$
\begin{aligned}
& \Delta v_{n, l}=v_{n+1, l}-v_{n, l} \propto \Delta v=\left(2 \int_{0}^{R} \frac{\mathrm{d} r}{C_{\mathrm{s}}}\right)^{-1}=\left(2 \int_{0}^{R} \frac{\mathrm{d} r}{\sqrt{\Gamma_{1} p / \rho}}\right)^{-1} \\
& \delta v_{n, l}=v_{n-1, l+2}-v_{n, l} \propto \frac{\Delta v}{v_{n, l}} \times r_{\mathrm{i}} \text { and } r_{\mathrm{i}}=\int_{0}^{R} \frac{\partial C_{\mathrm{s}}}{\partial r} \frac{\mathrm{d} r}{r}
\end{aligned}
$$

where $\Gamma_{1}, p$ and $\rho$ are the adiabatic exponent, pressure and density inside the star. Therefore $\Delta v$ is a measurement of the inverse of the time that acoustic waves, with a velocity $C_{\mathrm{s}}$, take to travel from the centre to the stellar surface. According to Kjeldsen \& Bedding (1995), for a fully ionised star composed of an ideal gas with an "average" temperature $\langle T\rangle \propto M^{2} / R^{4}$, one gets:

$\Delta v \propto \sqrt{M / R^{3}} \propto \sqrt{\langle\rho\rangle}=\sqrt{\frac{1}{4 / 3 \pi R^{3}} \int_{0}^{R} 4 \pi \rho(r) r^{2} \mathrm{~d} r}$

i.e. $\Delta v$ depends on a global stellar parameter $(\langle\rho\rangle)$. The sound speed gradient integral $r_{\mathrm{i}}$ (defined on Eq. (2)) is related to the asteroseismic ratio $r_{n, l}=\delta v_{n, l} / \Delta v_{n, l+1}$ (Roxburgh \& Vorontsov 2003). Equation (2) hints that $r_{\mathrm{i}}$ and $\delta v_{n, l}$ are sensitive to the sound speed gradient $\left(\partial C_{\mathrm{s}} / \partial r\right)$ near the stellar centre. Thus $\delta v_{n, l}$ is sensitive to the stellar structure and evolutionary status. Diagrams which compare these two frequency separations can be used to study solar-type stars (Christensen-Dalsgaard 1984). These are known as Christensen-Dalsgaard (C-D) diagrams.

\section{Evolution of the seismic properties}

Stellar models are required to analyse the evolution of the internal structure and seismic properties of PMS stars. PMS models produced in preparation for the COROT mission (available at http://www . astro.up.pt/corot/models) were used in this study. These models were produced using the CESAM stellar 

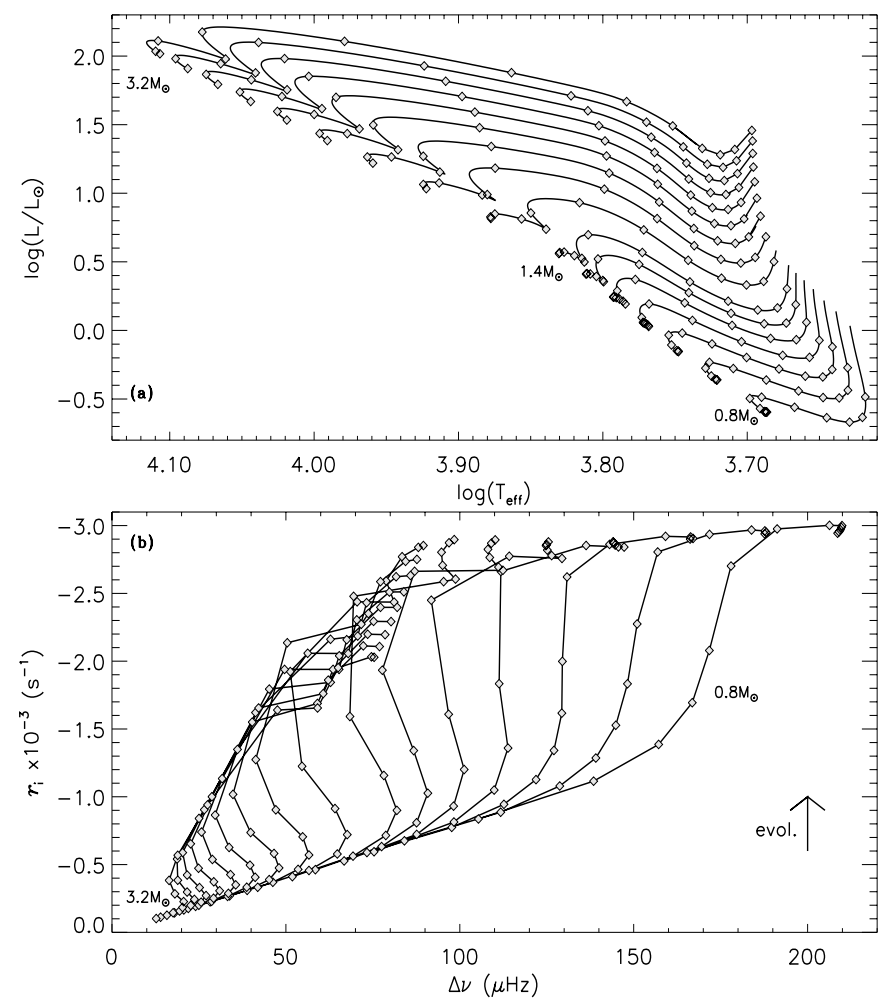

Fig. 1. a) PMS evolutionary tracks for 0.8 to $3.2 M_{\odot}$ stars. The diamonds correspond to the models described in Sect. 3. b) C-D diagram produced using the same models. The arrow indicates the direction in which evolution takes place.

evolutionary code (Morel 1997), applying the same procedures and assuming the same physical ingredients used in the modelling of EK Cep (Marques et al. 2004). These $Z=0.02$ models have masses between 0.8 and $3.2 M_{\odot}$. For each mass, 13 models were available. These are equally spaced in time, ranging from the end of the Hayashi track up to the ZAMS. Figure 1a shows each model's position in the HR diagram.

The model's seismic parameters $\left(\Delta v \& r_{\mathrm{i}}\right)$ were estimated using the integrals shown in Eqs. (1) and (2). The C-D diagram displayed in Fig. 1b was produced following Roxburgh \& Vorontsov's approach (2003), i.e. $r_{\mathrm{i}}$ was used instead of $\delta v_{n, l}$. The overlap of different models on the C-D diagram implies that this diagram is only useful to analyse stars with less than $1.3 M_{\odot}$.

\subsection{Sound speed gradient integral vs. effective temperature}

By comparing, for each model, the effective temperature $\left(T_{\text {eff }}\right)$ against its $r_{\mathrm{i}}$ integral (Fig. 2a) one breaks the small and large separation degeneracy displayed by some of the models (Fig. 1b). This means that unlike C-D diagrams, $T_{\text {eff }}$ vs. $r_{\mathrm{i}}$ diagrams can be used to study objects more massive than $1.3 M_{\odot}$. Yet Monte Carlo simulations point out that, for low mass stars $(M<$ $\left.1.3 M_{\odot}\right), T_{\text {eff }}$ vs. $r_{\mathrm{i}}$ diagrams can only achieve the precision of C-D diagrams if $T_{\text {eff }}$ is accurately known. For instance, a $3.5 \%$ accuracy in the mass determination is achieved either by knowing $r_{\mathrm{i}}$ and $\Delta v$ with a $5 \%$ precision or by knowing $r_{\mathrm{i}}$ (with that accuracy) and $T_{\text {eff }}$ with a $50 \mathrm{~K}$ uncertainty.

Figure 2 shows that the $r_{\mathrm{i}}$ integral evolves in a similar way as the average sound speed gradient computed between 0.25 and 0.33 stellar radii $\left(\left\langle\partial C_{\mathrm{s}} / \partial r\right\rangle_{R / 4-R / 3}=\frac{1}{R / 3-R / 4} \int_{R / 4}^{R / 3} \frac{\partial C_{\mathrm{s}}}{\partial r} \mathrm{~d} r\right)$. This hints at $r_{\mathrm{i}}$ 's dependence on the inner sound speed gradient.

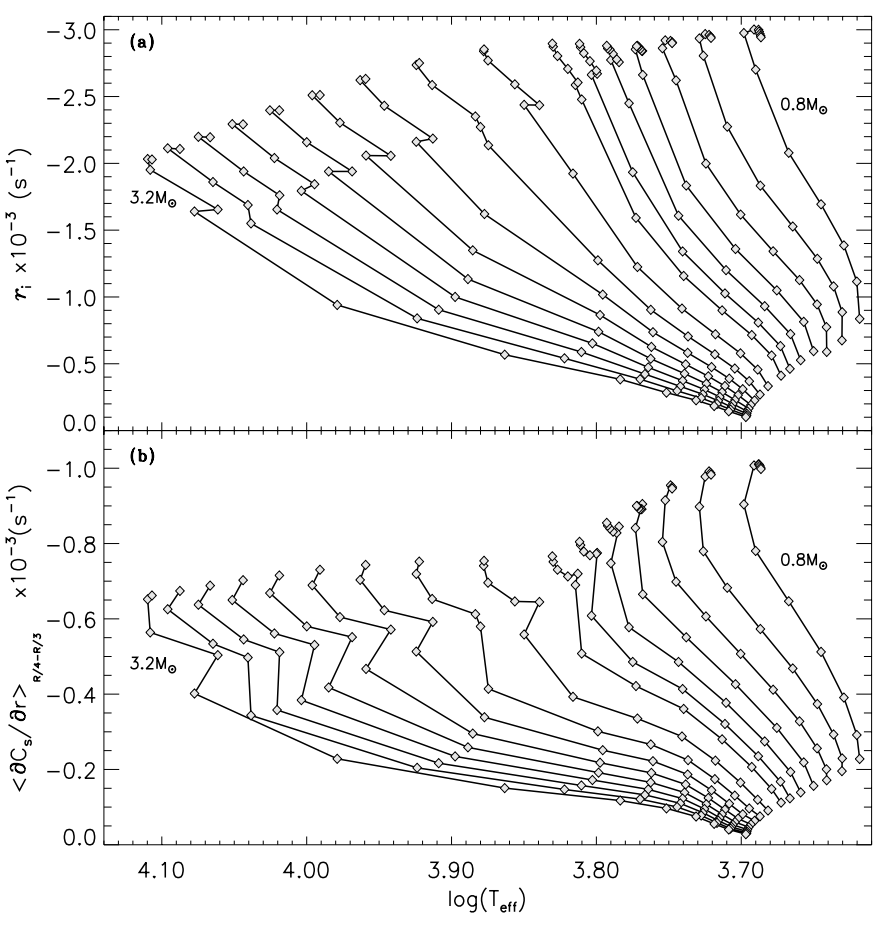

Fig. 2. a) Evolution of the sound speed gradient integral and b) the average sound speed gradient $\left\langle\partial C_{\mathrm{s}} / \partial r\right\rangle_{R / 4-R / 3}$ for the models displayed at Fig. 1 (diamonds).

\subsection{The large separation and the mean stellar density}

From Eq. (3) we get that $\Delta v \propto\langle\rho\rangle^{1 / 2}$. By relying on the definition of $\Delta v$ and $\langle\rho\rangle^{1 / 2}$ (Eqs. (1) and (2)) we can write the $\Delta v /\langle\rho\rangle^{1 / 2}$ ratio as:

$$
\begin{aligned}
& \Delta v /\langle\rho\rangle^{1 / 2}=\frac{1}{\frac{2}{C_{\mathrm{S}}(0)} \int_{0}^{R} \frac{C_{\mathrm{S}}(0)}{C_{\mathrm{S}}(r)} \mathrm{d} r} \times\left(\frac{4 / 3 \pi R^{3}}{\rho(0) \int_{0}^{R} 4 \pi \frac{\rho(r)}{\rho(0)} r^{2} \mathrm{~d} r}\right)^{0.5} \\
& \stackrel{x=\frac{r}{R}}{=}\left(\frac{C_{\mathrm{S}}(0)}{2 R \sqrt{3 \rho(0)}}\right) \times\left(\int_{0}^{1} \frac{C_{\mathrm{S}}(0)}{C_{\mathrm{S}}(x)} \mathrm{d} x\right)^{-1} \times\left(\int_{0}^{1} \frac{\rho(x)}{\rho(0)} x^{2} \mathrm{~d} x\right)^{-0.5} \\
& =K_{0} \times I_{C}{ }^{-1} \times I_{\rho}^{-0.5}
\end{aligned}
$$

where $K_{0}$ is an estimate of $\Delta v /\langle\rho\rangle^{1 / 2}$ obtained using the central density $(\rho(0))$ and sound speed $\left(C_{\mathrm{S}}(0)\right)$. The normalised sound speed $\left(I_{C}\right)$ and density $\left(I_{\rho}\right)$ integrals are independent of the model's radius, density and sound speed.

Figure 3 hints that changes in the density and sound speed profiles are partially responsible for the observed variations in the $\Delta v /\langle\rho\rangle^{1 / 2}$ ratio. Such profile changes are due to modifications in the relationship between pressure and density resulting from changes in the location/size of convective/ionisation regions that occur during the contraction towards the ZAMS.

This figure also shows that $\Delta v /\langle\rho\rangle>^{1 / 2}, K_{0}, I_{C}$ and $I_{\rho}$ are, to some extent, correlated with the model's effective temperature. Indeed, the dispersion of the model's $\Delta v /\langle\rho\rangle^{1 / 2}$ ratio in Fig. 3a is, at any given temperature, around 2 to $5 \%$ Thus knowing a star's effective temperature allows one to predict its $\Delta v /\langle\rho\rangle^{1 / 2}$ ratio.

\section{The contribution of metallicity}

Intermediate mass PMS stars are known for their $\delta$-Scuti type pulsations. Samadi et al. (2005) predicted the amplitudes of 

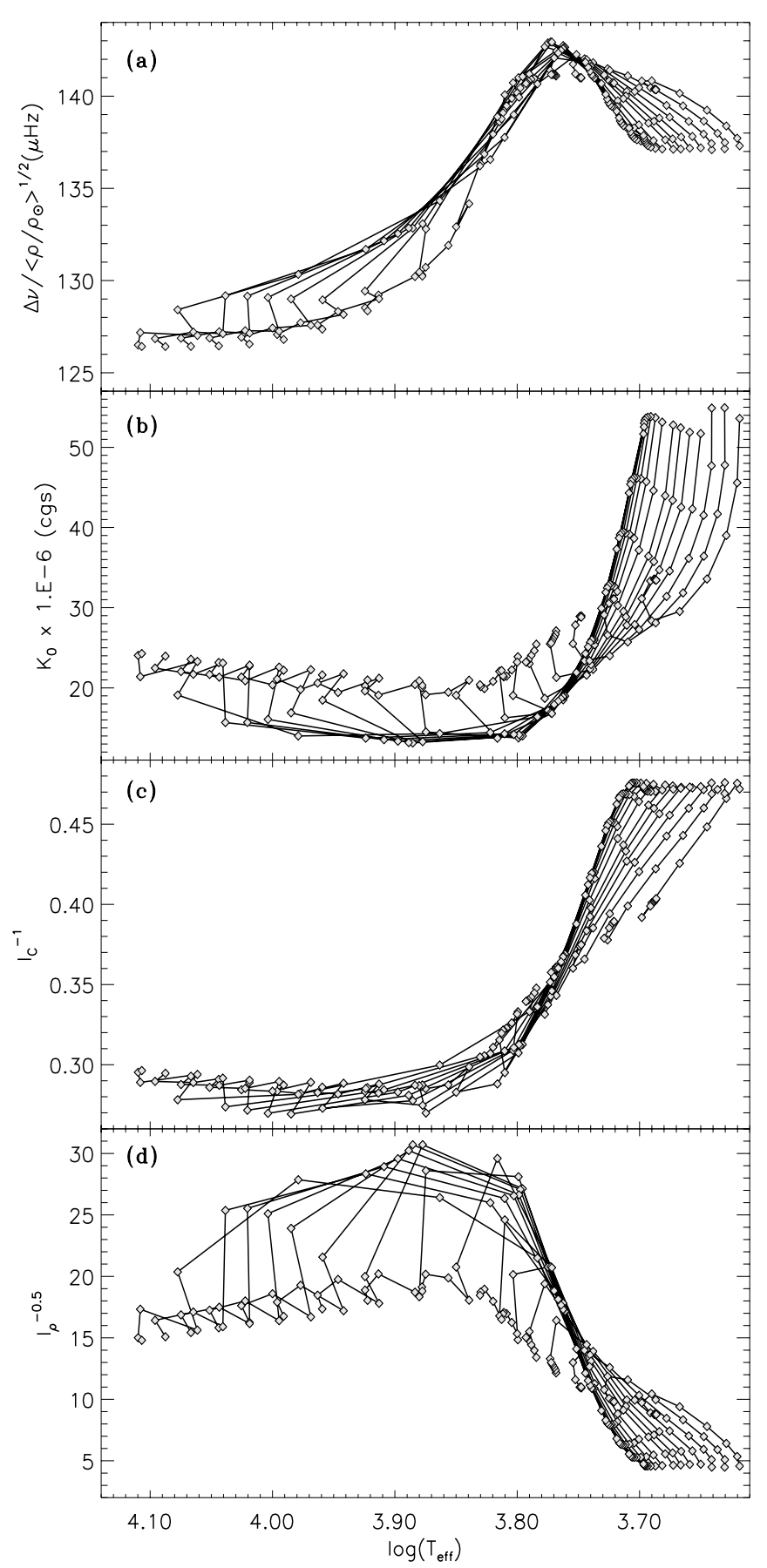

Fig. 3. a) Normalization of the large separation $(\Delta v)$ by the square root of the mean stellar density $\langle\rho\rangle^{1 / 2}$, b) $\Delta v /\langle\rho\rangle^{1 / 2}$ ratio estimated using the core density and sound speed, c) inverse of the profile integral $I_{C}$ and, d) inverse of the square root of the profile integral $I_{\rho}$ for the models described in Sect. 3 (diamonds).

solar-type pulsations for low mass PMS stars located to the right of the IS. The size (in solar radius) of the outer convective region of Samadi's PMS model \#9 is smaller than that of the $\log \left(T_{\text {eff }}\right) \leq 3.85$ models used in Sect. 3. Therefore, one may be tempted to extrapolate Samadi's result for $\log \left(T_{\text {eff }}\right) \leq 3.85$ stars. On the other hand, intermediate mass stars located near the ZAMS may not display solar-type pulsations since their outer convective layers are smaller (about one order of magnitude) than the one of Samadi's PMS model \#9. The convective cores of

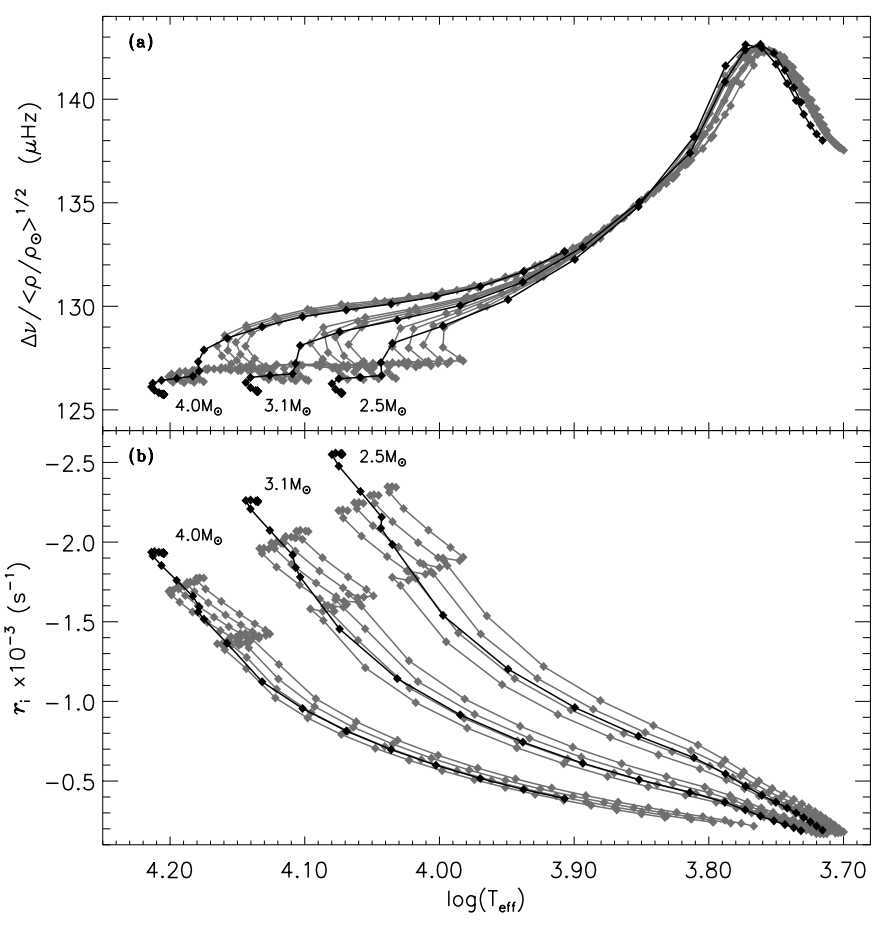

Fig. 4. a) Evolution of the $\Delta v /\langle\rho\rangle^{1 / 2}$ ratio and b) $r_{\mathrm{i}}$ integral for diferent stellar models. The black diamonds correspond to $2.5 M_{\odot}, 3.1 M_{\odot}$ and 4.0 $M_{\odot} Z=0.01$ models. The grey diamonds correspond to 2.5-2.8 $M_{\odot}$, 3.1-3.4 $M_{\odot}$ and 4.0-4.3 $M_{\odot} Z=0.02$ models.

A type stars $\left(\sim 2 M_{\odot}\right)$ can drive $g$ modes (Browning et al. 2004; Antonello et al. 2006). However, currently it is unknown whether core convection can drive solar-type pulsations like outer convection does. Nonetheless, such conjectures require an extrapolation of the study of solar-type pulsations to more massive stars.

In order to explore the metalicity effect on the seismic properties of intermediate mass stars, we selected $Z=0.01$ and $Z=0.02$ models produced by Marques to study the PMS $\delta$-Scuti star VV Ser (Ripepi et al. 2007). These models have masses ranging between 2.5 and $4.4 M_{\odot}$ covering, for each mass, 30 evolutionary stages equally spaced in time. Figure 4 shows that the seismic properties of $Z=0.02$ PMS stars evolve in a similar fashion to those of $Z=0.01$ stars. This happens because the internal structure of both types of stars evolves in the same way. However quantitatively there are some differences. Near the ZAMS, metal-poor stars are denser and have larger sound speed gradients than their $Z=0.02$ counterparts. Therefore their $r_{\mathrm{i}}$ integral is larger.

\section{Asteroseismic test of PMS models}

The seismic properties of a given star can be used to test stellar models that reproduce, within given uncertainties, its position in the HR diagram. Figure 5 shows this. In this exercise the target is a $2.8 M_{\odot}, Z=0.01$ star with $\log \left(L / L_{\odot}\right)=1.51$ and $\log \left(T_{\text {eff }}\right)=3.77$. The models tested here are the same ones used in Sect. 4. In this case a $20 \%$ uncertainty is assumed in the target's $\log \left(L / L_{\odot}\right)$, a $1 \%$ uncertainty in $\log \left(T_{\text {eff }}\right)$ and a $5 \%$ accuracy in its seismic parameters $\Delta v$ and $r_{\mathrm{i}}$. Figure 5 hints that, as expected from Eq. (3), $\Delta v$ puts constraints on the model's density. In the same manner we can see how $r_{\mathrm{i}}$ puts constraints on the stellar evolutionary status. Conversely, models that reproduce the seismic properties of a given star can constrain its global stellar parameters (e.g. Pinheiro et al. 2003). 


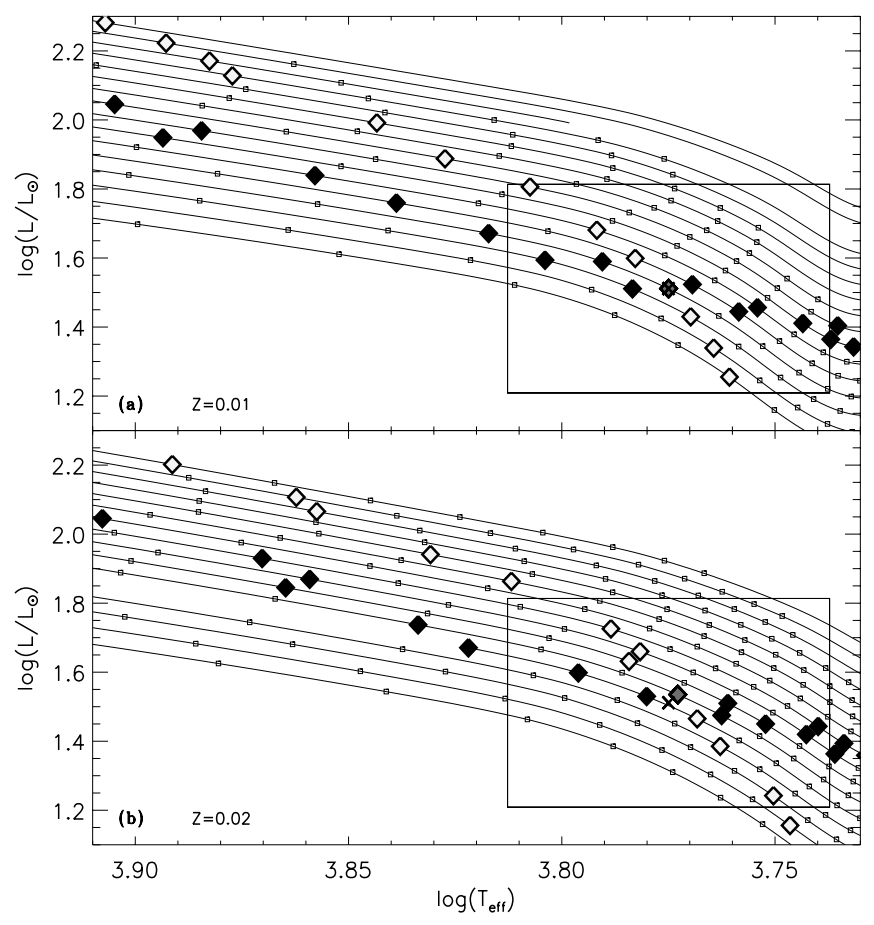

Fig. 5. a) $Z=0.01$ and, b) $Z=0.02$ stellar models that reproduce, with $5 \%$ accuracy, the large separation $\Delta v$ (black diamonds) and the $r_{\mathrm{i}}$ integral (white diamonds) of a $2.8 M_{\odot}, Z=0.01$ test star whose position in the HR diagram is given by the black cross $\left(\log \left(T_{\text {eff }}\right)=3.77\right.$ $\left.\& \log \left(L / L_{\odot}\right)=1.51\right)$. The grey diamonds correspond to the models that reproduce both seismic properties with a 5\% accuracy, while the small squares represent models that do not reproduce them. The boxes represent a $1 \% \times 20 \%$ uncertainty region (in $\log \left(T_{\text {eff }}\right)$ and $\log \left(L / L_{\odot}\right)$ ) around the black crosses. The lines are 2.5 to $4.0 M_{\odot}$ PMS evolutionary tracks.

Table 1. Models that occupy the same position in the HR diagram and have different seismic characteristics.

\begin{tabular}{|c|c|c|c|c|c|c|}
\hline$M / M_{\odot}$ & $\log \left(T_{\text {eff }}\right)$ & $\log \left(L / L_{\odot}\right)$ & $Z$ & age(Myr) & $\Delta v(\mu \mathrm{Hz})$ & $r_{\mathrm{i}}\left(\mathrm{s}^{-1}\right)$ \\
\hline 2.5 & 3.75 & 1.16 & 0.01 & 1.66 & 27.9 & -366.4 \\
\hline \multirow[t]{2}{*}{2.5} & 3.75 & 1.16 & 0.02 & 2.46 & 28.6 & -454.0 \\
\hline & & & \multicolumn{2}{|c|}{ relative difference } & $2.3 \%$ & $19.3 \%$ \\
\hline 2.6 & 3.86 & 1.68 & 0.01 & 2.25 & 24.1 & -762.4 \\
\hline \multirow[t]{2}{*}{2.7} & 3.86 & 1.68 & 0.02 & 2.74 & 24.4 & -799.1 \\
\hline & & & \multicolumn{2}{|c|}{ relative difference } & $1.3 \%$ & $4.6 \%$ \\
\hline 2.6 & 4.05 & 1.92 & 0.01 & 2.82 & 53.3 & -1919.7 \\
\hline \multirow[t]{2}{*}{3.1} & 4.05 & 1.92 & 0.02 & 2.58 & 59.2 & -1662.3 \\
\hline & & & \multicolumn{2}{|c|}{ relative difference } & $9.5 \%$ & $13.4 \%$ \\
\hline
\end{tabular}

According to Baglin et al. (2001), in a 150 day run COROT can achieve a $0.1 \mu \mathrm{Hz}$ accuracy in individual frequency determination. On the other hand, $\Delta v_{n, l} \approx 40 \mu \mathrm{Hz}$ and $\delta v_{n, l} \approx 6 \mu \mathrm{Hz}$ are typical frequency separations of intermediate mass PMS stars (Pinheiro et al. 2006). This means that COROT can determine $\Delta v_{n, l}$ and $\delta v_{n, l}$ with accuracies up to $0.5 \%$ and $4 \%$, respectively. Table 1 shows that some of the models used above have different seismic properties, despite occupying the same position in the HR diagram. Since these differences are larger that COROT's accuracy, one could use COROT to test these models.

\section{Conclusions and future developments}

The seismic characteristics of the PMS models evaluated here reflect their internal structure. Therefore, as the models contract towards the ZAMS their structure and seismic properties change. In general terms, the evolution of $\Delta v$ and $r_{\mathrm{i}}$ shows no significant dependence on the model's mass and metallicity. However these variations are correlated to some extent with the model's effective temperature. Indeed, in the range of metal abundances analysed here, the knowledge of star's effective temperature (with a $100 \mathrm{~K}$ uncertainty) allows us to infer its $\Delta v /\langle\rho\rangle^{1 / 2}$ ratio with a precision between 2 and $5.5 \%$.

Due to a degeneracy between the seismic properties $\left(\delta v \& r_{\mathrm{i}}\right)$ of some models, C-D diagrams are only useful for testing PMS models with less than $1.3 M_{\odot}$. This degeneracy is broken by taking into account their temperature. Consequently, $T_{\text {eff }}$ vs. $r_{\mathrm{i}}$ diagrams can be used to analyse stars more massive than $1.3 M_{\odot}$. On the other hand, the accuracy in individual frequency determination that COROT can achieve is enough to test several PMS evolutionary models. This result supports the study of young solartype pulsators.

In the near future we will analyse the effect that stellar parameters, such as the mixing and the overshooting, have on the seismic properties of low/intermediate mass PMS stars. The seismic properties expected for each model will be estimated using the ADIPLS pulsation code (Christensen-Dalsgaard 1982).

Acknowledgements. This work was supported by Fundação para a Ciência e a Tecnologia and FEDER (through POCI2010) through project POCI/CTE-AST/57610/2004. I would also like to thank M. Monteiro, J. Fernandes and the anonymous referee for their useful remarks and J. Marques for providing his models.

\section{References}

Antia, H. M. 2005, JApA, 26, 161

Antonello, E., Mantegazza, L., Rainer, M., \& Miglio, A. 2006, A\&A, 445, L15 Baglin, A., Auvergne, M., Catala, C., Michel, E., \& COROT Team 2001, ESASP 464, 395

Bedding, T. R., \& Kjeldsen, H. 2003, PASA, 20, 203

Breger, M. 1972, ApJ, 171, 539

Browning, M. K., Brun, A. S., \& Toomre, J. 2004, ApJ, 601, 512

Christensen-Dalsgaard, J. 1982, MNRAS, 199, 735

Christensen-Dalsgaard, J., \& Frandsen, S. 1983, SoPh, 82, 469

Christensen-Dalsgaard, J. 1984, Space Research in Stellar Activity and Variability, 11

Goldreich, P., \& Keeley, D. A. 1977, ApJ, 212, 243

Guenther, D. B. 2002, ApJ, 569, 911

Kjeldsen, H., \& Bedding, T. R. 1995, A\&A, 293, 87

Marconi, M., \& Palla, F. 1998, ApJ, 507, L141

Marques, J. P., Fernandes, J., \& Monteiro, M. J. P. F. G. 2004, A\&A, 422, 239

Mazumdar, A., Basu, S., Collier, B. L., \& Demarque, P. 2006, MNRAS, 372, 949

Monteiro, M. J. P. F. G., Christensen-Dalsgaard, J., \& Thompson, M. J. 2002, ESA SP-485, Stellar Structure and Habitable Planet Finding, 291-298

Morel, P. 1997, A\&AS, 124, 597

Pinheiro, F. J. G., Folha, D. F. M., Marconi, M., et al. 2003, A\&A, 399, 271-274

Pinheiro, F. J. G. 2006, 2005, Past Meets Present in A\&A, 23

Ripepi, V., \& Marconi, M. 2004, ESA SP-538: Stellar Structure and Habitable Planet Finding, 397

Ripepi, V., Bernabei, S., Marconi, M., et al. 2006, A\&A, 462, 1023

Roxburgh, I. W., \& Vorontsov, S. V. 2003, A\&A, 411, 215

Samadi, R., \& Goupil, M.-J. 2001, A\&A, 370, 136

Samadi, R., Goupil, M.-J., Alecian, E., et al. 2005, JApA, 26, 171

Tassoul, M. 1980, ApJS, 43, 469 